\title{
miR-22 Suppresses Tumor Invasion and Metastasis in Colorectal Cancer by Targeting NLRP3
}

This article was published in the following Dove Press journal:

Cancer Management and Research

\author{
Jinchun Cong ${ }^{1} *$ \\ Jian Gong ${ }^{2, *}$ \\ Chuanjia Yang ${ }^{3}$ \\ Zhixiu Xia' \\ Hong Zhang' \\ 'Department of Colorectal Tumor \\ Surgery, Shengjing Hospital of China \\ Medical University, Shenyang I 10004 , \\ People's Republic of China; ${ }^{2}$ Department \\ of Clinical Pharmacy, Shenyang \\ Pharmaceutical University, Shenyang \\ I 10016, People's Republic of China; \\ ${ }^{3}$ Department of General Surgery, \\ Shengjing Hospital of China Medical \\ University, Shenyang II0016, People's \\ Republic of China
}

*These authors contributed equally to this work
Correspondence: Hong Zhang

Email zhanghong7919@outlook.com
Purpose: This study aimed to investigate the effects of microRNA (miR)-22 on biological behaviors of colon cancer cells and to explore the relationship between miR-22 and NLRP3. Materials and Methods: First, human colon cancer HCT116 cells were transfected with a miR-22 mimic, miR-22 inhibitor, control mimic, and control inhibitor, respectively. CCK8, colony formation, and transwell assays were performed to observe cell proliferation, migration, and invasion. Western blotting was used to analyze the expression of recombinant NLRP3 (NLR family, pyrin domain-containing protein 3) and epithelial-mesenchymal transformation (EMT)-related proteins. The target relationship between miR-22 and NLRP3 was verified by double luciferase report. Second, an NLRP3 inhibitor and NLRP3 mimic were transfected into HCT116 cells, and the biological behaviors and EMT-related proteins were again observed. Finally, a nude mouse xenograft model was constructed to verify the above results.

Results: In vitro, compared with the control group, administration of the miR-22 mimic significantly decreased proliferation, migration, and invasion of HCT116 cells, whereas the miR-22 inhibitor markedly increased their proliferation and invasion $(p<0.05)$. Levels of NLRP3, interleukin-1 $\beta$ (IL-1 $\beta$ ), matrix metalloproteinase-9 (MMP-9), MMP-2, N-cadherin, and vimentin were significantly reduced after miR-22 mimic transfection $(p<0.05)$. Furthermore, silencing of NLRP3, a downstream gene of miR-22 in HCT116 cells, suppressed proliferation, migration, and invasion of HCT116 cells. However, overexpression of NLRP3 weakened the effects of the miR-22 mimic. In vivo, overexpression of miR-22 slowed the growth rate of tumors and reduced Ki-67 expression in tumor tissues compared with the model group $(\mathrm{p}<0.05)$. In tumor tissues, overexpression of miR-22 also decreased expression of NLRP3, IL-1 $\beta$, MMP-9, MMP-2, N-cadherin, and vimentin compared with the model group $(\mathrm{p}<0.05)$. Overexpression of NLRP3 weakened the role of miR-22 overexpression in vivo.

Conclusion: miR-22 suppresses cell proliferation, migration, and invasion in colorectal cancer by targeting NLRP3.

Keywords: miR-22, NLRP3, colorectal cancer, epithelial-mesenchymal transformation, invasion

\section{Introduction}

Colorectal cancer (CRC) is the third most commonly occurring cancer and is prevalent worldwide, especially in the developed world. ${ }^{1,2}$ According to the World Health Organization's GLOBOCAN database, there are more than 1.8 million new cases every year. ${ }^{3} \mathrm{CRC}$ is caused by the cumulative transformation of epithelial cells in the surface of the intestinal tract to cancerous cells. ${ }^{4,5}$ The survival rate for $\mathrm{CRC}$ after surgery has been less than $60 \%$ in recent years. ${ }^{6,7}$ 
Therefore, there is an urgent need to understand the biological mechanisms of CRC in order to develop new therapies. In the past decade, tumor-derived human cell lines have been a cornerstone of cancer research and have guided our understanding of the process of cancer development.

MicroRNAs (miRNAs) are small non-coding RNAs with a mean length of 21-25 nucleotides (nt). ${ }^{8}$ Previous research has demonstrated that miRNAs have an essential role in the post-transcriptional regulation of gene and noncoding RNA expression, thereby controlling signaling events, cell migration, proliferation, and various cellular pathways. ${ }^{9,11}$ Therefore, miRNA expression profiles are related to tumor progression and are valuable in the clinical diagnosis and prognosis of most cancers. Human chromosome 17p13.3 encodes a 22-nt miRNA, miR-22. The expression of miR-22 has been reported to be downregulated in different cancer lines, and it has been shown to function as a tumor suppressor in pancreatic cancer and breast cancer. ${ }^{12}$ It has also been shown to interrupt tumor progression via effects on proliferation, migration, and invasion, and could be used to control symptoms of breast cancer and cervical cancer. ${ }^{12}$ Moreover, the expression level of miR-22 is lower in CRC tissues than in normal tissues, and the progression of CRC was found to be blocked by silencing of hypoxia-inducible factor $1 .{ }^{13}$ However, further investigation of the mechanisms of miR-22 in CRC are needed.

NLRP3 (recombinant NLR family, pyrin domaincontaining protein 3 ) is recruited in response to pathogenic or endogenous signals and is responsible for maturation and secretion of pro-inflammatory cytokines including interleukin-1 $\beta$ (IL-1 $\beta$ ) and IL-18. ${ }^{14}$ Studies have shown that miR-22 binds directly to the $3^{\prime}$ untranslated region (3'-UTR) of NLRP3 to suppress cell proliferation in oral squamous cell carcinoma ${ }^{15}$ and gastric cancer. ${ }^{16}$ Nevertheless, the effects of miR-22 on epithelial-mesenchymal transformation (EMT) in CRC require further elucidation.

In this study, we designed control and experimental groups to confirm the function of miR-22 in human colon cancer cells. We also further verified that miR-22 represses EMT by targeting NLRP3 in CRC.

\section{Materials and Methods}

\section{Cell Culture}

Human colonic epithelial cells (HCoEpiC) and human colon cancer cell lines HCT116, HCT8, HT29, LS174T,
LOVO, and SW480 were all obtained from the Shanghai Institute of Cell Research, CAS. Cells were cultured with DEGM (Gibco) with 1\% penicillin-streptomycin and 10\% fetal bovine serum (FBS) at $37^{\circ} \mathrm{C}, 5 \% \mathrm{CO} 2$. Subcultured cells in the logarithmic growth phase were selected for further study.

\section{Cell Grouping and Transfection}

HCT116 cells were used for the following cell experiments. According to a previously reported method, ${ }^{15}$ cells were transfected with a miR-22 mimic and miR-22 inhibitor for $72 \mathrm{~h}$ to explore the function of miR-22. Lentiviral particles were constructed by Shanghai Jikai Biotechnology Co., Ltd. Cells were randomly divided into five groups: blank control (BC) group (no treatment), miR-22 overexpression negative control (NC1) group (transfected with miR-22 scramble), miR-22 overexpression (miR-22) group (transfected with miR-22 mimic), miR-22 silencing negative control (NC2) group (transfected with miR-22 inhibitor negative control), and miR-22 silencing (si-miR) group (transfected with miR22 inhibitor).

To further confirm that NLRP3 was a downstream gene of miR-22 in CRC, six groups were used: BC, miR-22, NLRP3 silencing negative control (NC3) group (transfected with NLRP3 short interfering RNA (siRNA) negative control vector), NLRP3-silencing (si-NLRP3) group (transfected with NLRP3 siRNA vector), miR-22 overexpression+NLRP3 overexpression negative control (miR +NC4) group (transfected with miR-22 mimic and NLRP3 mimic negative control), and miR-22 overexpression+NLRP3 overexpression (miR+NLRP3) group (transfected with miR-22 mimic and NLRP3 mimic).

\section{RT-PCR}

Cells were collected and centrifuged at $4{ }^{\circ} \mathrm{C}(12,000 \mathrm{rpm})$ for 5 min. Total RNA was extracted using TRIzol reagent according to the manufacturer's instructions (Tamara, Dalian, China). First-strand cDNA synthesis was performed with a cDNA Reverse Transcription Kit (Applied Biosystems, Waltham, MA, USA). RT-PCR (MasterCycler) consisted of 35 cycles of $95^{\circ} \mathrm{C}$ for $15 \mathrm{~s}, 60^{\circ} \mathrm{C}$ for $60 \mathrm{~s}$, and $72^{\circ} \mathrm{C}$ for $40 \mathrm{~s}$. Data were analyzed by the comparison $\mathrm{Ct}$ $\left(2^{-\Delta \Delta \mathrm{Ct}}\right)$ method and expressed as fold change relative to glyceraldehyde 3-phosphate dehydrogenase (GAPDH) or U6.

The primers were as follows.

miR-22 Forward: 5'-GCATGGAAGCTGCCAGTTG AAG-3' 
Reverse: 5'-ATCCAGTGCAGGGTCCGAGG-3'

U6 Forward: 5'-CTCGCTTCGGCAGCACA-3'

Reverse: 5'-AACGCTTCACGAATTTGCGT-3'

NLRP3 Forward: 5'-CCATCGGCAAGACCAAGA-3'

Reverse: 5'-ACAGGCTCAGAATGCTCATC-3'

GAPDH Forward: 5'-TGACTTCAACAGCGACACC

CA-3'

Reverse: 5'-CACCCTGTTGCTGTAGGCCAAA-3'

\section{CCK-8 Assay}

Cell viability was assessed using a Cell Counting Kit-8 (CCK8) assay kit (Dojindo, Japan) according to the manufacturer's protocol. Cells were cultured in 96-well plates at a density of $2 \times 10^{4}$ cells per mL. At $72 \mathrm{~h}, 10 \mu \mathrm{L}$ of CCK-8 solution was added to plates, followed by incubation for $4 \mathrm{~h}$ at $37^{\circ} \mathrm{C}$. Optical density was measured at 450 $\mathrm{nm}$ to reflect cell viability.

\section{Colony Formation Assay}

Cells in the logarithmic growth phase were detached with $0.25 \%$ trypsin, and the cell concentration was adjusted to 250 cells $/ \mathrm{mL}$. These cells were cultured in a six-well plate at $37^{\circ} \mathrm{C}$ with $5 \% \mathrm{CO}_{2}$ for $2-3$ weeks; the fresh medium was changed every 3 days. Cells were then fixed in methanol, and $1 \mathrm{~mL}$ Giemsa working fluid was added to each well for $30 \mathrm{~min}$. Cells were washed with water, and excess water was removed with filter paper.

\section{Transwell Assays}

Cell invasion and migration were examined by transwell assay. For the invasion assay, the upper chamber of the filter was pre-coated with pre-cooled DMEM and Matrigel (Solebao, Beijing) at 1:1 dilution, and $5 \times 10^{4}$ transfected cells were cultured in the upper chamber at $37^{\circ} \mathrm{C}$ for $4 \mathrm{hrs}$. Next, $600 \mu \mathrm{L}$ of DMEM with $10 \%$ FBS was added to the lower chamber and cultured at $37^{\circ} \mathrm{C}$ with $5 \% \mathrm{CO}_{2}$ for 72 hrs. After washing twice with phosphate-buffered saline, the chambers were fixed in $5 \%$ glutaraldehyde at $4{ }^{\circ} \mathrm{C}$, then stained with $0.1 \%$ crystal violet (Solarbio, Beijing, China) for 30 mins. For the migration assay, cells were cultured in the upper chamber using pre-cooled DMEM without Matrigel. Finally, the numbers of cells were observed at 400x magnification by an inverted microscope (Olympus, Japan).

\section{Western Blotting}

Supernatants of cells or tissues were collected, and BCA kits (Solarbio, Beijing, China) were used to measure protein concentration. Cell suspensions were gently mixed with $10 \%$ SDS-PAGE at $95^{\circ} \mathrm{C}$ for 5 mins. Proteins were transferred to polyvinylidene fluoride membranes for $30 \mathrm{~min}$ and blocked with $5 \%$ bovine serum albumin (BSA) for $1 \mathrm{~h}$. The membranes were incubated with primary antibodies including matrix metalloproteinase-2 (MMP-2) (1:500, orb193343), MMP-9 (1:500, orb227878), E-cadherin (1:500, orb43407), N-cadherin (1:500, orb227888), vimentin $(1: 500, \quad$ orb229187), NLRP3 (1:500, orb319065), IL-1 $\beta$ (1:500, orb339111), and $\beta$-actin (1:2000, orb178392) at $4^{\circ} \mathrm{C}$ overnight. All antibodies were from Biorbyt (Shanghai, China). After warming, proteins were further incubated with anti-rabbit IgG secondary antibody (1:1000, ABIN101998, antibodies-online, Aachen, Germany) for $1 \mathrm{~h}$ and visualized using enhanced chemiluminescence for 3-5 min. Protein expression was normalized by $\beta$-actin. Grayscale scanning and quantification were performed using the Image $\mathrm{J}(\mathrm{NIH})$ software.

\section{Luciferase Reporter Assay}

The wild-type or mutant 3'-UTR of NLRP3 for miR-22 was amplified in a pGL3/luciferase vector (Promega, Madison, WI, USA) and cloned into cells downstream of the luciferase gene. The luciferase activity of cells was analyzed by dual-luciferase assay (Promega) $48 \mathrm{~h}$ after transfection.

\section{Xenografts Models in Nude Mice}

Twenty-four male BALB/c nude mice, weighing 16-18 g, were purchased from Beijing Vital River Laboratory Animal Technology Co., license number SCXK (Beijing) 20160006. All mice were fed at $24-26^{\circ} \mathrm{C}$. The animal experiments followed National Institutes of Health guidelines (NIH Pub. No. 85-23, revised 1996) and were approved by the Animal Protection and Use Committee of Shengjing Hospital of China Medical University (No. 20190612015). HCT116 cells in the logarithmic growth phase were digested with $0.25 \%$ trypsin and adjusted to a concentration of $5 \times 10^{7} / \mathrm{mL} .{ }^{15}$ A $0.1-\mathrm{mL}$ cell dilution was injected into the skin of the back of the right forelimb. The 24 nude mice were randomly divided into four groups: model group (injected with untreated HCT116 cells), miR22 group (injected with HCT116 cells transfected with miR-22 mimic), si-NLRP3 group (injected with HCT116 cells transfected with NLRP3 siRNA), and miR+NLRP3 group (injected with HCT116 cells transfected with miR22 mimic and NLRP3 mimic). 


\section{Tumor Volume}

Tumor volumes were calculated based on the long diameter (L) and short diameter (W) of the tumor every 7 days using the following formula: tumor volume $(\mathrm{V})=$ (long diameter $\mathrm{x}$ short diameter $\left.{ }^{2}\right) / 2$. After 28 days, mice were sacrificed using an intraperitoneal injection of $0.6 \%$ sodium pentobarbital $(40 \mathrm{mg} / \mathrm{kg})$ and tumor tissues were weighed. The tumor specimens were partially fixed in $4 \%$ paraformaldehyde and stored at $-80^{\circ} \mathrm{C}$.

\section{Immunohistochemical Assay}

Tumor tissues were fixed with $4 \%$ paraformaldehyde and cut into $4-\mu \mathrm{m}$ sections. Three percent $\mathrm{H}_{2} \mathrm{O}_{2}$ methanol solution was added for $20 \mathrm{~min}$, followed by the addition of citrate buffer ( $\mathrm{pH} \mathrm{6.0)}$ for $10 \mathrm{~min}$ at $95^{\circ} \mathrm{C}$. Samples were blocked with 5\% BSA for $20 \mathrm{~min}$. Polyclonal rabbit anti-human Ki67 antibody (1:200, orb88614, Biorbyt, Cambridge, UK) was added and incubated at $4{ }^{\circ} \mathrm{C}$ overnight. After rewarming, samples were incubated with goat anti-rabbit horseradish peroxidase-conjugated IgG antibody (1:1000, ABIN101988, antibodies-online). Tissues were colored with $\mathrm{DAB}$, then counterstained, dehydrated, cleared, and sealed, before being observed at 400x magnification (Olympus, Japan) and analyzed with the Aperio ImageScope 11.1 software.

\section{Statistical Analysis}

Data were processed using SPSS 19.0 and presented as mean \pm standard deviation. Statistical significance was determined using analysis of variance for multiple comparisons followed by Tukey's test. $\mathrm{p}<0.05$ was considered statistically significant.

\section{Results}

\section{miR-22 Overexpression Suppressed Cell Proliferation, Migration, and Invasion in Human Colon Cancer Cells}

To assess the function of miR-22 in human colon cancer cells, miR-22 expression levels in different cells were analyzed (Figure 1A). Compared with HCoEpiC cells, miR-22 levels were decreased in human colon cancer cells $(\mathrm{p}<0.05)$. We chose HCT116 cells for further investigation of the effects of miR-22 because they had the lowest miR-22 levels among the cancer cells tested. Different expression of miR-22 in HCT116 cells was established (Figure 1B). HCT116 cell activities were tested at $72 \mathrm{~h}$ by CCK-8 assay (Figure 1C); the results showed that miR-22 overexpression significantly suppressed cell activities, whereas silencing of miR-22 promoted cell activities compared with the control group $(p<0.05)$. Cell proliferation was also analyzed by plate clone formation test (Figure 1D). Colony formation numbers were significantly increased after the administration of the miR-22 inhibitor and decreased after miR-22 mimic transfection compared with the control group $(\mathrm{p}<0.05)$. Similarly, cell invasion and migration were increased in the si-miR group but decreased in the miR-22 group compared with the control group (Figure $1 \mathrm{E}, \mathrm{p}<0.05$ ). Taken together, these results confirm the essential role of miR-22 in maintaining proliferation, invasion, and migration of HCT116 cells.

\section{miR-22 Overexpression Restrained EMT in HCTII 6 Cells}

Western blotting was performed to determine the levels of MMP-2, MMP-9, N-cadherin, E-cadherin, and vimentin in each group (Figure 2). The results showed that the expression levels of MMP-2, MMP-9, vimentin, and N-cadherin were markedly reduced, but E-cadherin expression was significantly increased, in the miR-22 group compared with the control group $(\mathrm{p}<0.05)$. Levels of MMP-2, MMP-9, vimentin, and N-cadherin were increased, and levels of E-cadherin were decreased compared with the control group $(\mathrm{p}<0.05)$. These data suggest that miR-22 has an essential role in the regulation of EMT in HCT116 cells.

\section{miR-22 Targeted NLRP3 in HCTI I 6 Cells}

NLRP3 has been reported to be involved in the metastasis of many tumor types. The expression of NLRP3 in HCT116 cells was detected by quantitative RT-PCR (qRT-PCR) and Western blotting (Figure 3A and B). The expression of NLRP3 was significantly decreased in the miR-22 group but significantly increased in the simiR group compared with the control group $(p<0.05)$. The expression of IL-1 $\beta$ showed a similar trend to that of NLRP3 (Figure 3B). As shown in Figure 3C, a target binding site between miR-22 and NLRP3 was identified by TargetScan and miRanda. The dual-luciferase reporter assay confirmed that NLRP3 is a target gene of miR-22 (Figure 3D). These results indicate that miR-22 targets NLRP3 to regulate the biological behaviors of HCT116 cells. 

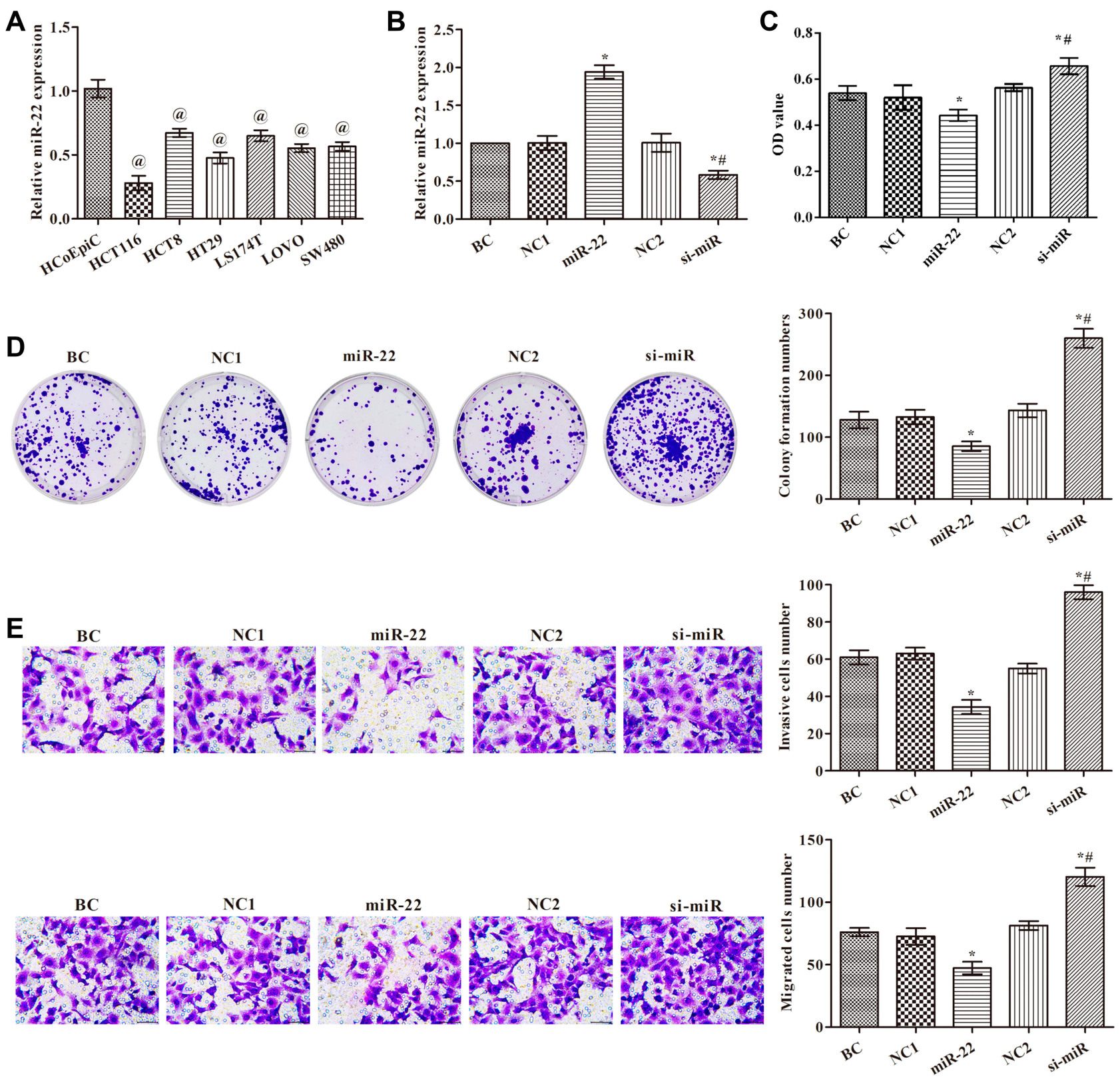

Figure I Effects of miR-22 on cell proliferation, migration, and invasion in human colon cancer cells. (A) miR-22 levels examined by qRT-PCR in cells; (B) miR-22 levels examined by qRT-PCR in transfected HCTI I6 cells; (C) cell proliferation examined by CCK8; (D) cell proliferation examined by colony formation assay; (E) cell invasion and migration examined by transwell assay $(\times 400)$. ${ }^{\mathrm{p}}<0.05$ compared with $\mathrm{HCoEpiC}$ group; ${ }^{*} \mathrm{p}<0.05$ compared with $\mathrm{BC}$ group; ${ }^{*} \mathrm{p}<0.05$ compared with miR-22 group.

Abbreviations: BC, blank control group, without any treatment; NCI, miR-22 overexpression negative control group, transfected with miR-22 scramble; miR-22, miR-22 overexpression group, transfected with miR-22 mimic; NC2, miR-22 silencing negative control group, transfected with miR-22 inhibitor negative control; si-miR, miR-22 silencing group, transfected with miR-22 inhibitor.

\section{miR-22 Overexpression Inhibited}

\section{HCTII 6 Cell Proliferation, Migration, and Invasion via Targeting NLRP3}

To further verify the results presented above, we divided the cells into six groups. The expression of NLRP3 mRNA in each group is shown in Figure 4A. The data showed that the NLRP3 mimic and siRNA were successfully transfected. The effects of miR-22 on proliferation via regulation of NLRP3 in HCT116 cells were studied. The cells' abilities and colony formation numbers were significantly reduced in the si-NLRP 3 and miR-22 groups, but the NLRP3 mimic weakened the effects of the miR-22 mimic ( $p<0.05$, Figure $4 B$ and $C$ ). In the transwell assay (Figure 4D), transfection of miR-22 mimic or NLRP3 siRNA markedly decreased invasion and migration of 

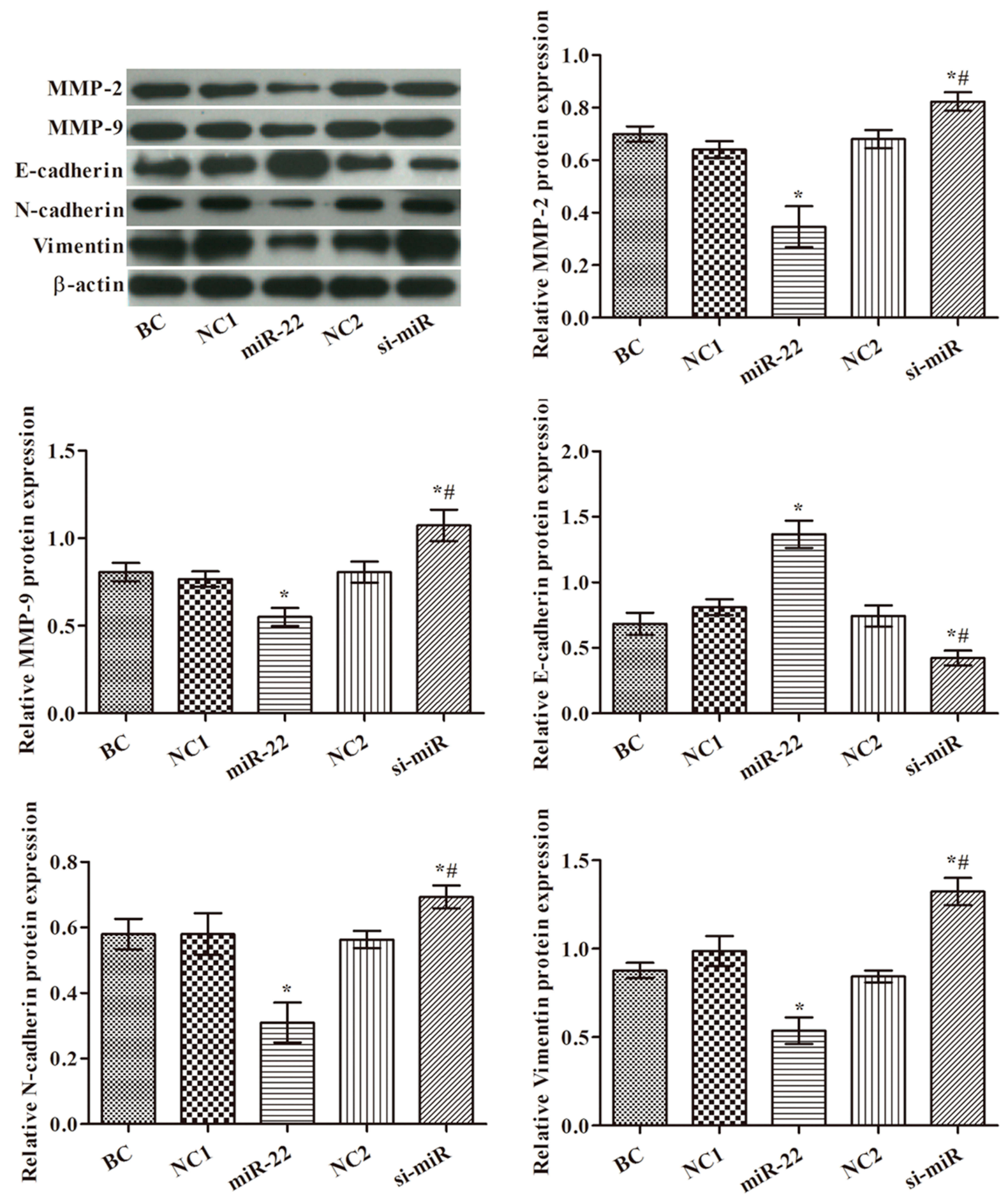

Figure 2 Effects of miR-22 on levels of MMP-2, MMP-9, E-cadherin, N-cadherin and vimentin in HCTII6 cells. Western blot images are representative of three replicates. ${ }^{*}<0.05$ compared with BC group; ${ }^{p} \mathrm{p}<0.05$ compared with miR-22 group.

HCT116 cells, and transfection of the NLRP3 mimic reduced the effects of the miR-22 mimic $(\mathrm{p}<0.05)$. This suggests that miR-22 overexpression inhibited HCT116 cell proliferation, migration, and invasion via regulating NLRP3.
miR-22 Overexpression Controlled EMT via Regulating NLRP3 in HCTII 6 Cells

To determine whether miR-22 overexpression controlled EMT via targeting NLRP3 in HCT116 cells, we examined the expression of related proteins using Western blotting 


\section{A}
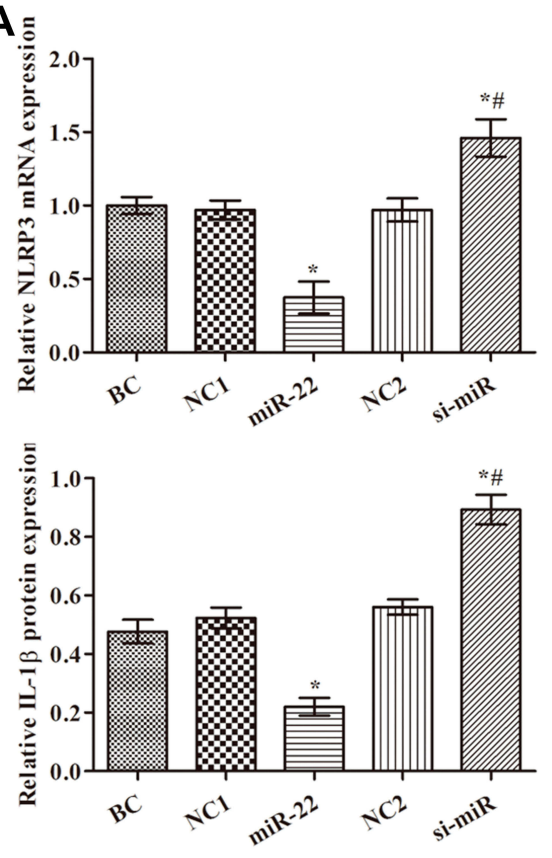

B

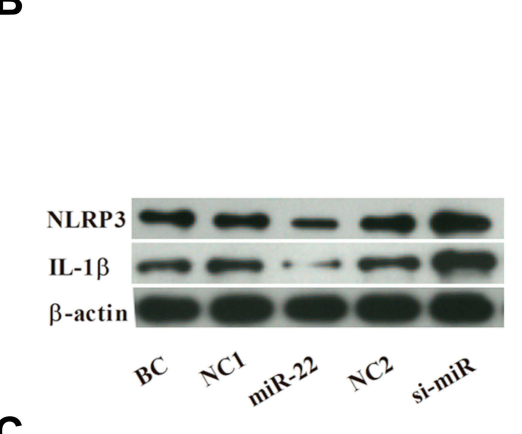

C

NLRP3 WT 5'-guugaaaUUỤu-UGGCAGCUa-3' miR-22 3'-ugucaagAAGuUgACCGUCGAa-5 NLRP3 MUT 5'-guugaaauuuua-GTTACTAGa-3'
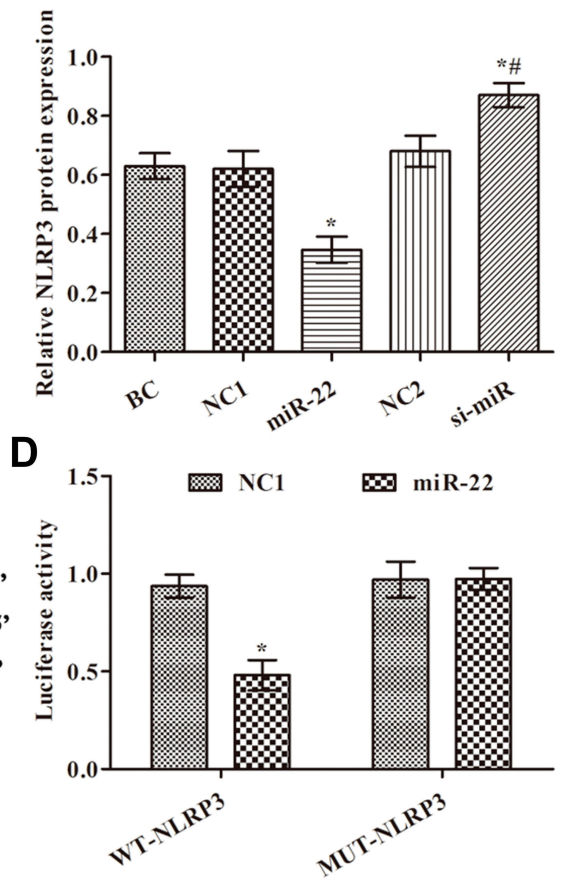

Figure 3 Effects of miR-22 on expression of NLRP3 in HCTII6 cells. (A) NLRP3 levels examined by RT-PCR in each group; (B) NLRP3 and IL-I $\beta$ levels examined by Western blotting; $(\mathbf{C})$ prediction of binding site between miR-22 and the $3^{\prime}$ untranslated regions of NLRP3 by TargetScan and miRanda; (D) dual-luciferase reporter gene assay. ${ }^{*}<0.05$ compared with $B C$ group; ${ }^{*}<0.05$ compared with miR-22 group.

(Figure 5). The results indicated that the expression levels of NLRP3, IL-1 $\beta$, MMP-2, MMP-9, vimentin, and N-cadherin proteins were lower in both the miR-22 and si-NLRP3 groups compared with the $\mathrm{BC}$ group $(\mathrm{p}<0.05)$. However, the expression of E-cadherin protein was greatly increased in the miR22 and si-NLRP3 groups compared with the BC group $(p<0.05)$. Thus, it is clear that NLRP3 overexpression weakened the inhibition of the miR-22 mimic.

\section{miR-22 Overexpression Repressed Tumor Growth via Regulating NLRP3 in Nude Mice}

To further confirm whether miR-22 inhibited tumorigenesis in vivo, we conducted a tumor formation experiment in nude mice using HCT116 cells (Figure 6). In comparison to the model group, tumor size and weight were suppressed in the other groups $(\mathrm{p}<0.05$, Figure $6 \mathrm{~A}$ and B). Compared with the miR-22 mimic group, tumor volume and weight were obviously increased in the miR +NLRP3 group $(\mathrm{p}<0.05)$. Ki-67 expression in tumor tissue was analyzed by immunohistochemistry (Figure 6C). The percentage of Ki67-positive cells in the tumor tissues was decreased in the miR-22 and si-NLRP3 groups. Compared with the miR-22 group, the percentage of Ki67-positive cells in tumor tissue was increased in the miR+NLRP3 group $(\mathrm{p}<0.05)$. These results indicate that miR-22 overexpression repressed tumor growth via regulating NLRP3.

\section{miR-22 Overexpression Suppressed EMT by Targeting NLRP3 in Nude Mice}

Consistent with the results of the in vitro experiments, the expression levels of NLRP3, IL-1 $\beta$, MMP-9, MMP-2, vimentin, and $\mathrm{N}$-cadherin proteins were decreased, but that of E-cadherin protein was increased, in all groups compared with the model group (Figure 7, $<<0.05$ ). Compared with the miR-22 group, NLRP3, IL-1 $\beta$, MMP-9, MMP-2, vimentin, and $\mathrm{N}$-cadherin, and E-cadherin protein expression levels were all decreased in the miR+NLRP3 group $(p<0.05)$.

\section{Discussion}

Previous studies have reported potential functions of miR-22 in various cancers, including $\mathrm{CRC}^{17}$ and osteosarcoma. ${ }^{18}$ In CRC, miR-22 suppresses cell proliferation and migration in vitro and in vivo by targeting HuR. ${ }^{17}$ In osteosarcoma, miR-22 inhibits biological functions and increases cisplatin sensitivity via targeting S100A11. ${ }^{18}$ Here, we examined the function of miR-22 in CRC and found that it had a tumorinhibiting role, consistent with the results of other studies. We also showed that miR-22 repressed CRC cell proliferation, migration, and invasion in vitro and in vivo. The 
A

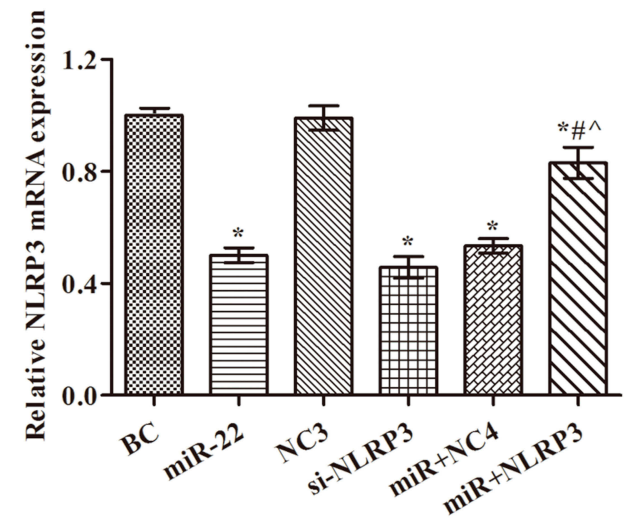

C

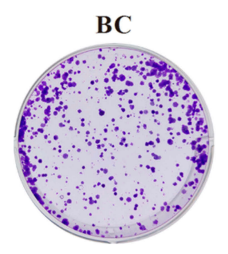

si-NLRP3
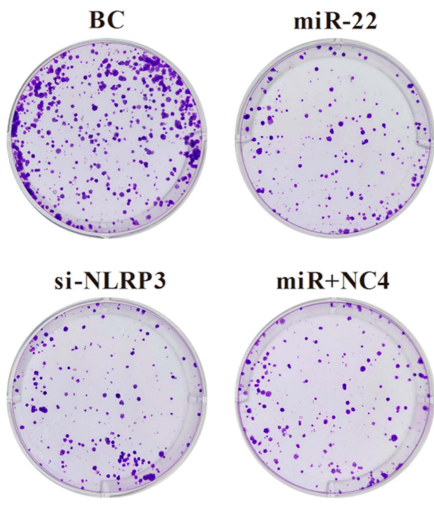

$\mathrm{miR}+\mathrm{NC4}$
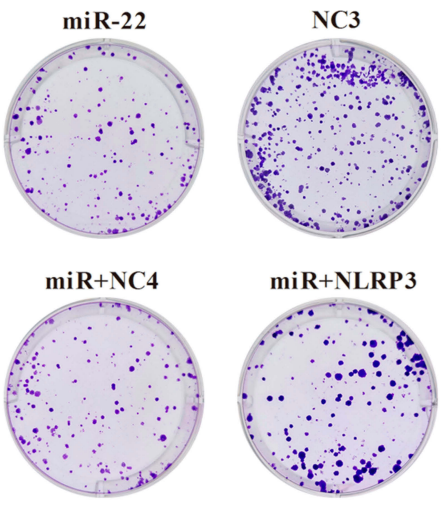

NC3

D

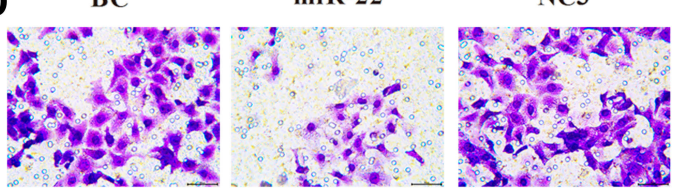

si-NLRP3

miR+NC4

miR+NLRP3
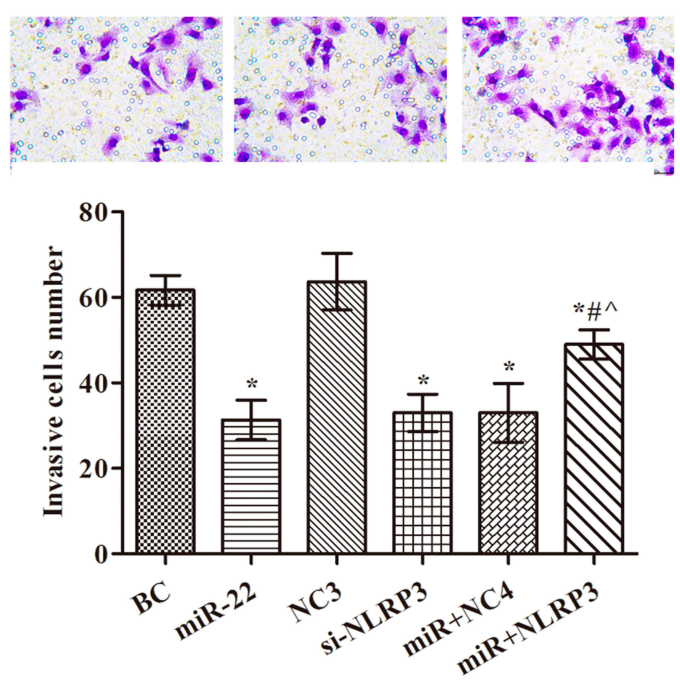

B
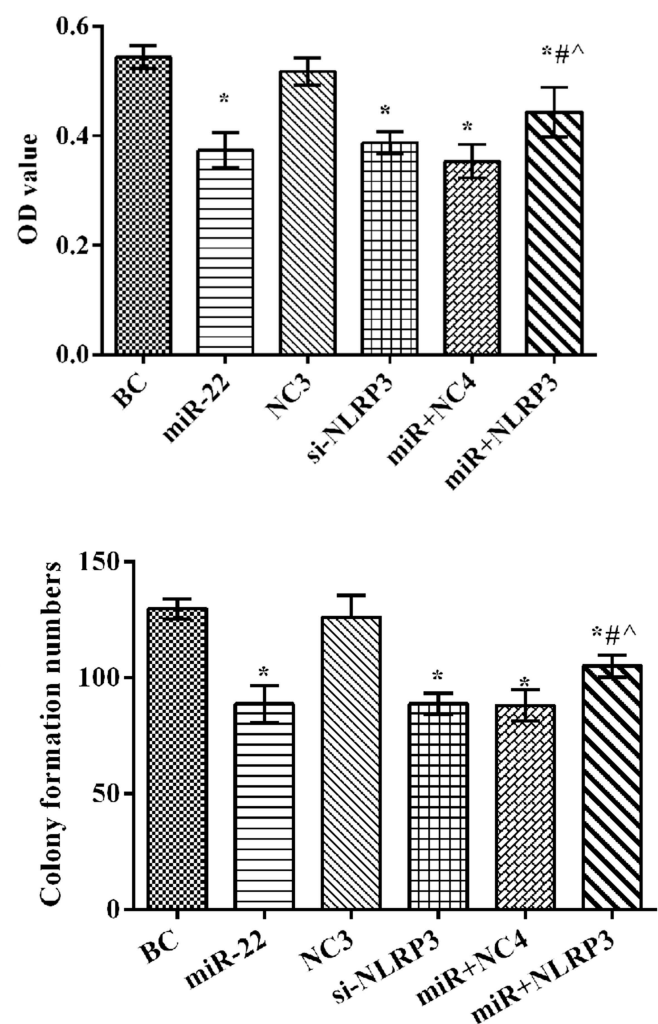

BC

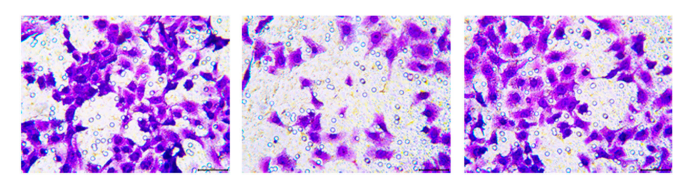

si-NLRP3

$\mathrm{miR}+\mathrm{NC} 4$

miR+NLRP3
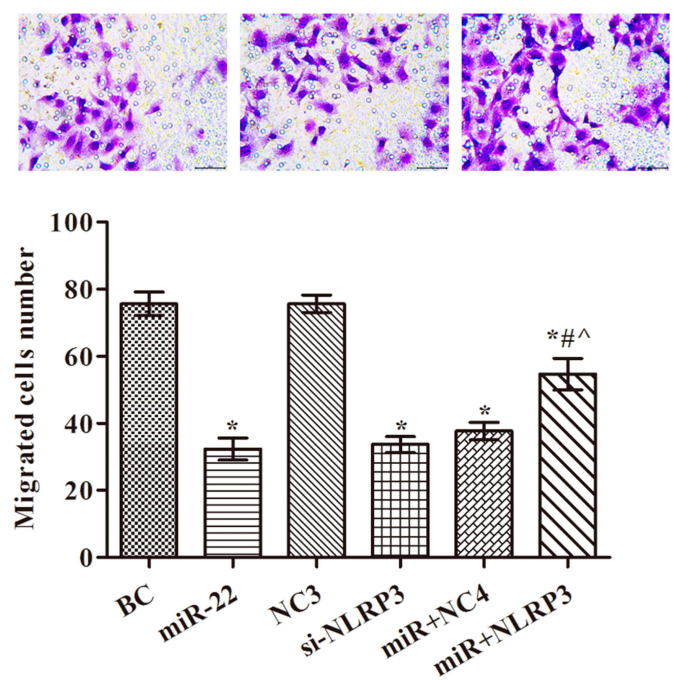

Figure 4 miR-22 overexpression inhibited proliferation, migration, and invasion in HCTII6 cells via targeting NLRP3. (A) NLRP3 levels examined by RT-PCR in each group; (B) cell proliferation examined by CCK8 assay; (C) cell proliferation examined by colony formation assay; (D) cell invasion and migration examined by transwell assays ( $\times 400) .{ }^{*} p<0.05$ compared with BC group; ${ }^{*} \mathrm{p}<0.05$ compared with miR-22 group; ${ }^{\wedge} \mathrm{p}<0.05$ compared with si-NLRP3 group.

Abbreviations: NC3, NLRP3 silencing negative control group, transfected with NLRP3 siRNA negative control vector; si-NLRP3, NLRP3 silencing group, transfected with NLRP3 siRNA vector; miR+NC4, miR-22 overexpression+NLRP3 overexpression negative control group, transfected with miR-22 mimic and NLRP3 mimic negative control; miR+NLRP3, miR-22 overexpression+NLRP3 overexpression group, transfected with miR-22 mimic and NLRP3 mimic. 


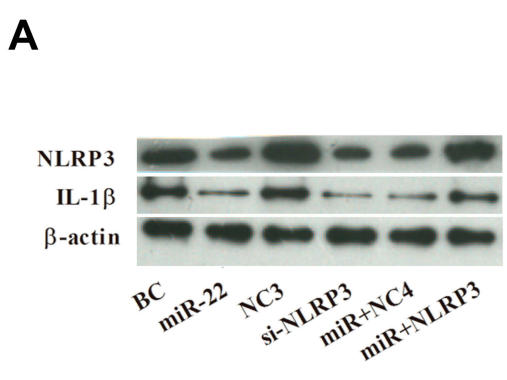

B
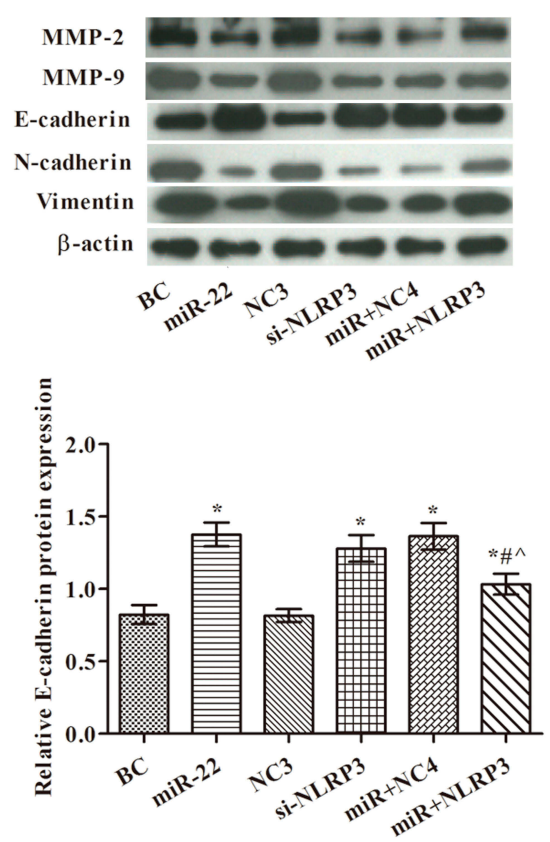
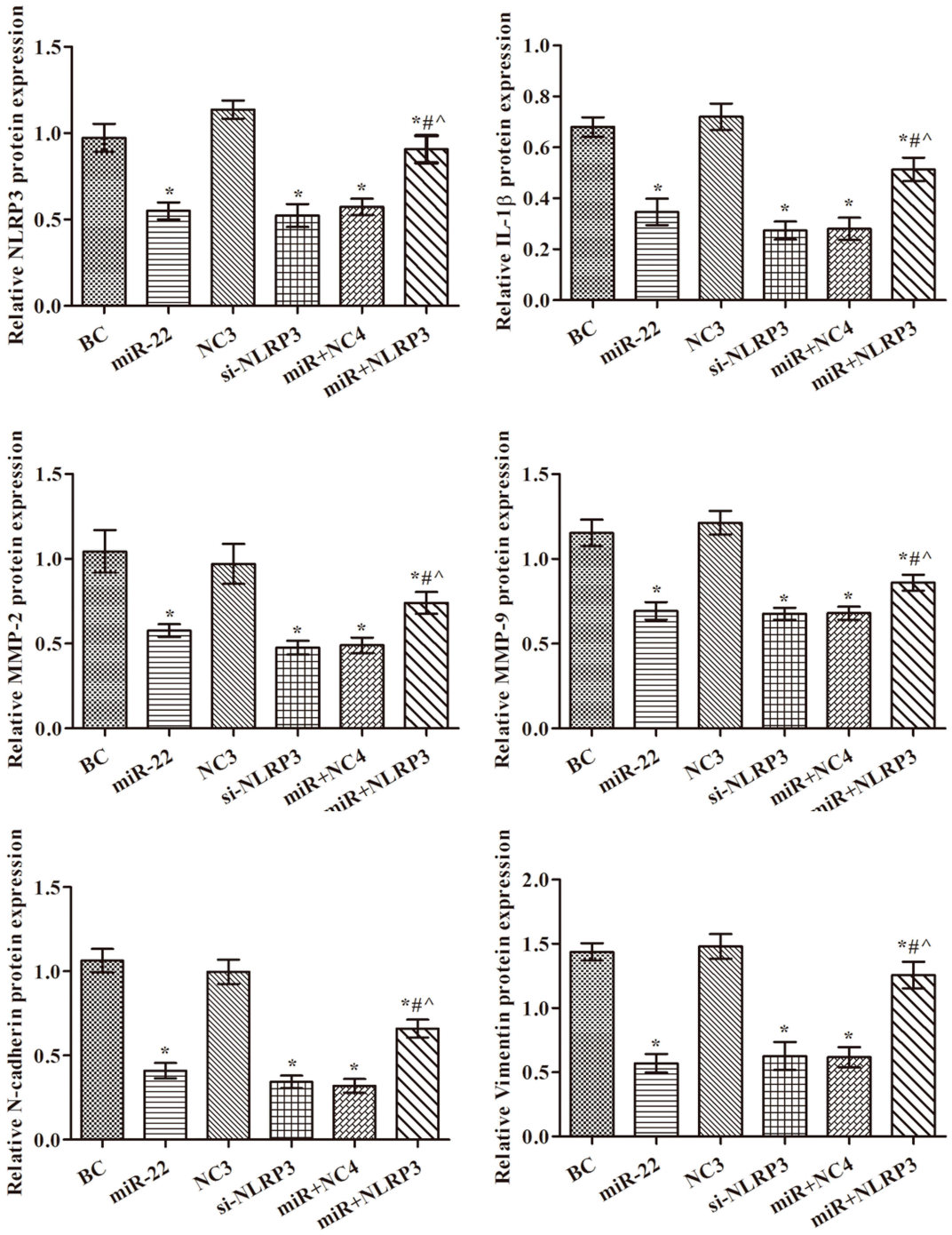

Figure 5 miR-22 overexpression suppressed EMT-related protein expression via targeting NLRP3 in HCTII6 cells. (A) NLRP3 and IL-I $\beta$ levels examined by Western blotting in each group; (B) MMP-2, MMP-9, E-cadherin, N-cadherin, and vimentin levels examined by Western blotting in each group. Western blot images are representative of three replicates. ${ }^{*} \mathrm{p}<0.05$ compared with BC group; ${ }^{\#} \mathrm{p}<0.05$ compared with miR-22 group; ${ }^{\wedge} \mathrm{p}<0.05$ compared with si-NLRP3 group.

underlying mechanisms by which miR-22 regulates tumor progression are diverse and complex, and are worth exploring in more detail in CRC.

The immune system resists to other erosion via the expression of pattern recognition receptors (PRRs), which have been linked to the function of various inflammasomes. ${ }^{19}$ Members of the NLR (nucleotidebinding domain and leucine-rich repeat-containing) protein family are important PRRs with a crucial role. ${ }^{20}$ The NLRP3 inflammasome plays an important part in activating the inflammasome modulated innate immune function, ${ }^{21,22}$ leading to activation of the IL-1 $\beta$ family of cytokines. Recent reports have verified that excessive activation of the NLRP3 inflammasome enhances metastasis of various tumor types, including $\mathrm{CRC} .{ }^{23,24}$ In the current study, NLRP3 was poorly expressed when miR22 was overexpressed but highly expressed when miR22 was silenced, indicating that miR-22 effectively repressed the expression of NLRP3. Dual-luciferase reporter assays confirmed that NLRP3 was a target gene of miR-22.

In CRC, NLRP3 is associated with tumor size, invasion, lymph node metastasis, and TNM stage, potentially via activating the EMT process. ${ }^{24}$ MMPs are a major group of proteases known to regulate the turnover of the extracellular matrix and are associated with chronic inflammation. ${ }^{25}$ To further investigate the effects of miR-22 on MMPs and EMT in CRC via targeting NLRP3, we also examined the expression of MMP-9, MMP-2, vimentin, N-cadherin, and E-cadherin. Overexpression of miR-22 reduced levels of 

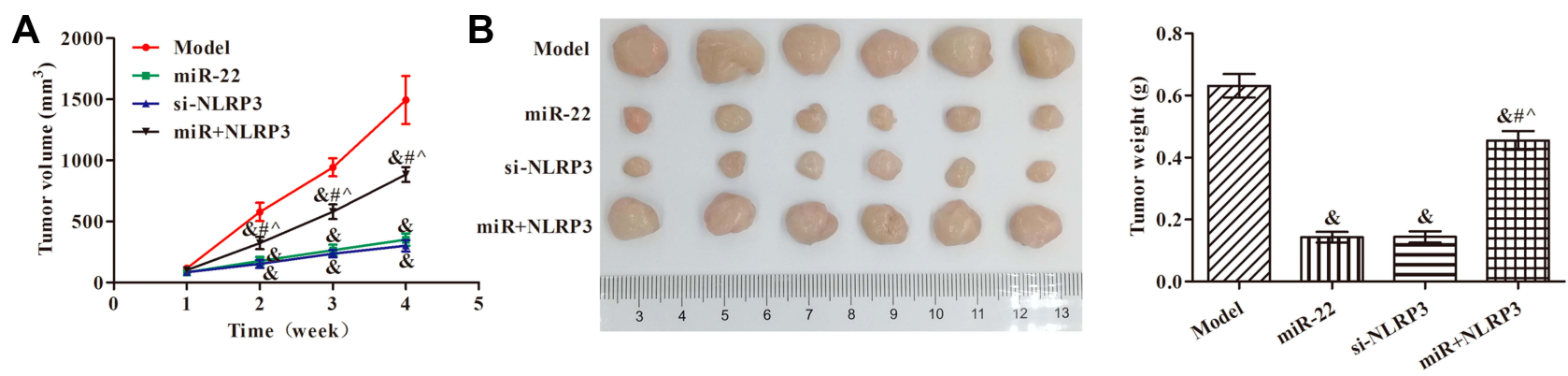

C
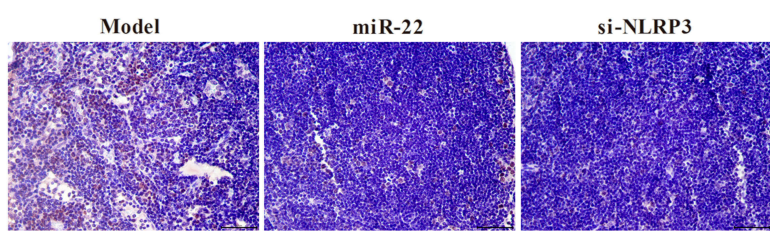

$\operatorname{miR}+\mathrm{NLRP} 3$
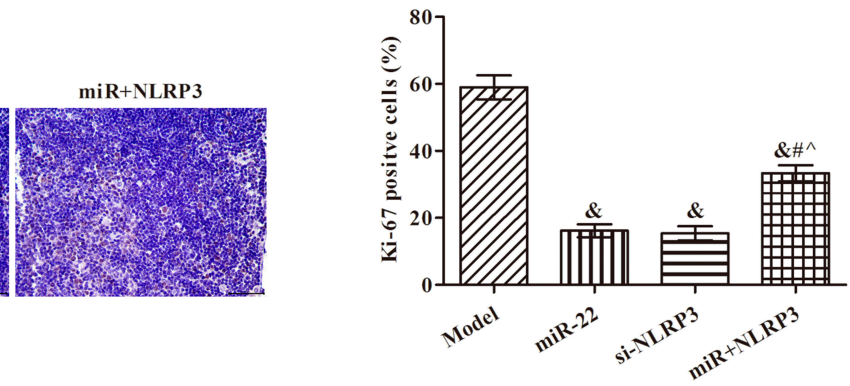

Figure 6 Effects of miR-22 on the growth of HCTII 6 cell xenografts via targeting NLRP3. (A) Tumor volumes; (B) tumor weights; (C) immunohistochemistry to detect Ki67 expression in tumor tissues $(\times 400)$. ${ }^{2} \mathrm{p}<0.05$ compared with model group; ${ }^{*} \mathrm{p}<0.05$ compared with miR-22 group; ${ }^{\wedge}<0.05$ compared with si-NLRP3 group.

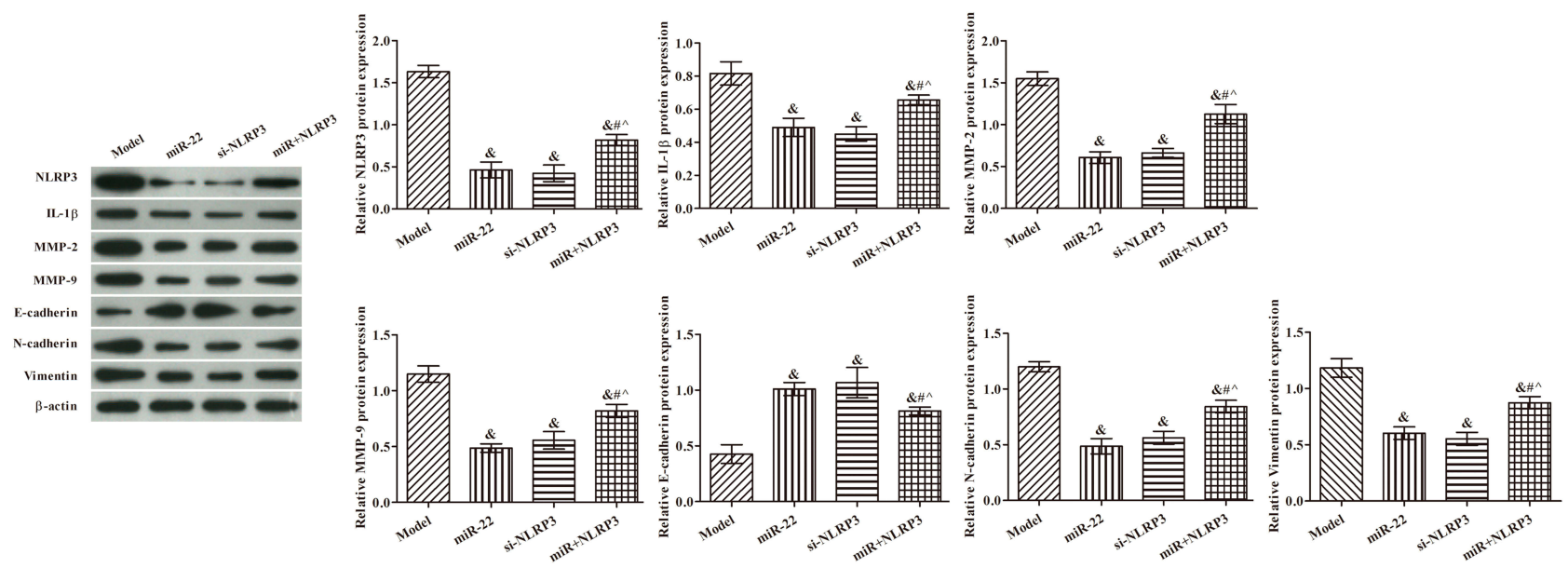

Figure 7 Effects of miR-22 on expression of related proteins in HCTI 16 cell xenografts via targeting NLRP3. Western blot images are representative of three replicates. $\&_{p}<0.05$ compared with model group; ${ }^{\#} p<0.05$ compared with miR-22 group; $\hat{p}<0.05$ compared with si-NLRP3 group.

MMP-9, MMP-2, vimentin, and $\mathrm{N}$-cadherin proteins via regulation of NLRP3.

Although the above results demonstrate that miR-22 suppresses colon cancer invasion and metastasis by targeting NLRP3, the study had many limitations and deficiencies. Only HCT116 cells were used for the experiments; the study should be replicated using other CRC cells. Levels of secreted IL-1 $\beta$ in supernatants should be analyzed using, for example, ELISA assays. Furthermore, the effects of stimulation of cells with IL-1 $\beta$ or lipopolysaccharide should be studied in order to compare the NLRP3 overexpression of cells.

\section{Conclusions}

In summary, miR-22 has a tumor-suppressing role in $\mathrm{CRC}$, and overexpression of miR-22 inhibits migration, EMT, and growth in vitro and in vivo by regulating NLRP3 expression. These findings provide new insights into the underlying mechanisms of CRC progression and highlight miR-22 as a potential prognostic biomarker and therapeutic target. 


\section{Acknowledgment}

We thank Charlesworth Author Services for editing this manuscript.

\section{Funding}

This study was supported by the Natural Science Foundation Guidance Program of Liaoning Province (2019-ZD-0747).

\section{Disclosure}

The authors declare that they have no conflicts of interest.

\section{References}

1. Ferlay J, Shin HR, Bray F, et al. Estimates of worldwide burden of cancer in 2008: GLOBOCAN 2008. Int $J$ Cancer. 2010;127 (12):2893-2917. doi:10.1002/ijc.25516

2. Nixon J, Newbold P, Mustelin T, Anderson GP, Kolbeck R. Monoclonal antibody therapy for the treatment of asthma and chronic obstructive pulmonary disease with eosinophilic inflammation. Pharmacol Ther. 2017;169:57-77. doi:10.1016/j.pharmthera.2016. 10.016

3. Ferlay J, Colombet M, Soerjomataram I, et al. Estimating the global cancer incidence and mortality in 2018: GLOBOCAN sources and methods. Int $J$ Cancer. 2019;144(8):1941-1953. doi:10.1002/ ijc. 31937

4. Armaghany T, Wilson JD, Chu Q, Mills G. Genetic alterations in colorectal cancer. Gastrointest Cancer Res. 2012;5(1):19-27.

5. Testa U, Pelosi E, Castelli G. Colorectal cancer: genetic abnormalities, tumor progression, tumor heterogeneity, clonal evolution and tumor-initiating cells. Med Sci (Basel). 2018;6(2):31.

6. Denlinger CS, Barsevick AM. The challenges of colorectal cancer survivorship. J Natl Compr Canc Netw. 2009;7(8):883-893; quiz 894. doi:10.6004/jncen.2009.0058

7. Lithner M, Klefsgard R, Johansson J, Andersson E. The significance of information after discharge for colorectal cancer surgery-a qualitative study. BMC Nurs. 2015;14:36. doi:10.1186/ s12912-015-0086-6

8. Ling H, Fabbri M, Calin GA. MicroRNAs and other non-coding RNAs as targets for anticancer drug development. Nat Rev Drug Discov. 2013;12(11):847-865. doi:10.1038/nrd4140

9. O'Brien J, Hayder H, Zayed Y, Peng C. Overview of microRNA biogenesis, mechanisms of actions, and circulation. Front Endocrinol (Lausanne). 2018;9:402. doi:10.3389/fendo.2018.00402

10. Peng Y, Croce CM. The role of microRNAs in human cancer. Signal Transduct Target Ther. 2016;1:15004. doi:10.1038/sigtrans.2015.4
11. Lan $\mathrm{H}$, Lu H, Wang X, Jin H. MicroRNAs as potential biomarkers in cancer: opportunities and challenges. Biomed Res Int. 2015; 2015:125094. doi:10.1155/2015/125094

12. Wang J, Li Y, Ding M, et al. Molecular mechanisms and clinical applications of miR-22 in regulating malignant progression in human cancer (Review). Int J Oncol. 2017;50(2):345-355. doi:10.3892/ ijo.2016.3811

13. Li X, Nie J, Mei Q, Han WD. MicroRNAs: novel immunotherapeutic targets in colorectal carcinoma. World J Gastroenterol. 2016;22 (23):5317-5331. doi:10.3748/wjg.v22.i23.5317

14. Tezcan G, Martynova EV, Gilazieva ZE, et al. MicroRNA post-transcriptional regulation of the NLRP3 inflammasome in immunopathologies. Front Pharmacol. 2019;10:451. doi:10.3389/ fphar.2019.00451

15. Feng X, Luo Q, Wang H, Zhang H, Chen F. MicroRNA-22 suppresses cell proliferation, migration and invasion in oral squamous cell carcinoma by targeting NLRP3. J Cell Physiol. 2018;233 (9):6705-6713. doi:10.1002/jcp.26331

16. Li S, Liang X, Ma L, et al. MiR-22 sustains NLRP3 expression and attenuates $\mathrm{H}$. pylori-induced gastric carcinogenesis. Oncogene. 2018;37(7):884-896. doi:10.1038/onc.2017.381

17. Liu Y, Chen X, Cheng R, et al. The Jun/miR-22/HuR regulatory axis contributes to tumourigenesis in colorectal cancer. Mol Cancer. 2018;17(1):11. doi:10.1186/s12943-017-0751-3

18. Zhou X, Natino D, Zhai X, Gao Z, He X. MicroRNA22 inhibits the proliferation and migration, and increases the cisplatin sensitivity, of osteosarcoma cells. Mol Med Rep. 2018;17(5):7209-7217. doi:10. 3892/mmr.2018.8790

19. Olive C. Pattern recognition receptors: sentinels in innate immunity and targets of new vaccine adjuvants. Expert Rev Vaccines. 2012;11 (2):237-256. doi:10.1586/erv.11.189

20. Carneiro LA, Fritz JO, Kufer TA, et al. NLRs: nucleotide-binding domain and leucine-rich-repeat-containing proteins. EcoSal Plus. 2009;3(2). doi:10.1128/ecosalplus.8.8.3.

21. Davis BK, Wen H, Ting JP. The inflammasome NLRs in immunity, inflammation, and associated diseases. Annu Rev Immunol. 2011;29:707-735. doi:10.1146/annurev-immunol-031210-101405

22. Mangan MSJ, Olhava EJ, Roush WR, et al. Targeting the NLRP3 inflammasome in inflammatory diseases. Nat Rev Drug Discov. 2018;17(8):588-606. doi:10.1038/nrd.2018.97

23. Chung IC, OuYang CN, Yuan SN, et al. Pretreatment with a heat-killed probiotic modulates the NLRP3 inflammasome and attenuates colitis-associated colorectal cancer in mice. Nutrients. 2019;11(3):516. doi:10.3390/nu11030516

24. Shao X, Lei Z, Zhou C. NLRP3 promotes colorectal cancer cell proliferation and metastasis via regulating epithelial mesenchymal transformation. Anticancer Agents Med Chem. 2020;20. doi:10.21 74/1871520620666200220112741

25. Robert S, Gicquel T, Victoni T, et al. Involvement of matrix metalloproteinases (MMPs) and inflammasome pathway in molecular mechanisms of fibrosis. Biosci Rep. 2016;36(4). doi:10.1042/ BSR20160107.
Cancer Management and Research is an international, peer-reviewed open access journal focusing on cancer research and the optimal use of preventative and integrated treatment interventions to achieve improved outcomes, enhanced survival and quality of life for the cancer patient.
The manuscript management system is completely online and includes a very quick and fair peer-review system, which is all easy to use. Visit http://www.dovepress.com/testimonials.php to read real quotes from published authors. 